\title{
New method of porous Ge layer fabrication: structure and optical properties
}

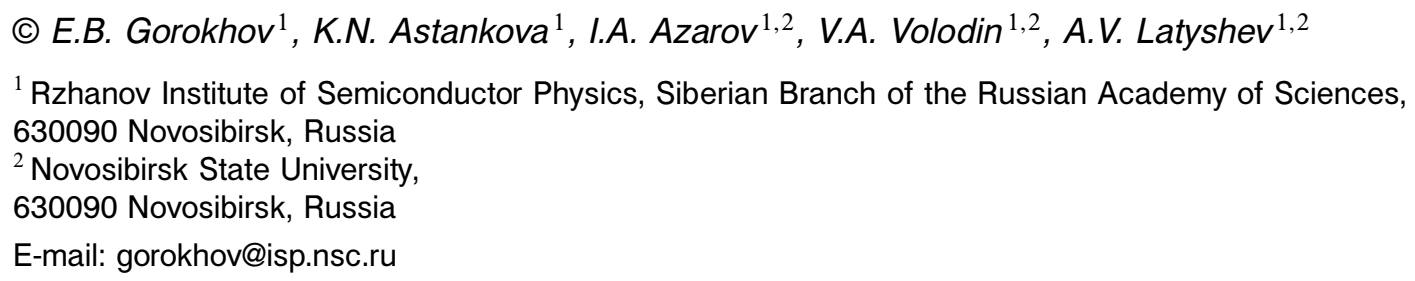

Porous germanium films were produced by selective removal of the $\mathrm{GeO}_{2}$ matrix from the $\mathrm{GeO}_{2}\langle\mathrm{Ge}-\mathrm{NCs}\rangle$ heterolayer in deionized water or HF. On the basis of Raman and infrared spectroscopy data it was suppose $\langle\mathrm{Ge}-\mathrm{NCs}\rangle$ heterolayers. The kinetics of air oxidation of amorphous porous Ge layers was investigated by scanning ellipsometry. Spectral ellipsometry allowed estimating the porosity of amorphous and crystalline porous Ge layers, which was $\sim 70 \%$ and $\sim 80 \%$, respectively.

\section{Acknowledgement}

Ellipsometry and Raman measurements were funded by the Russian Foundation for Basic Research (project No 16-07-00975). SEM and IR spectroscopy investigations were carried out with the financial support of the Russian Science Foundation (project No 14-22-00143). 